\title{
Enseñanza de la Electrostática por Medio de la Construcción de Prototipos de Bajo Costo y el Aprendizaje Basado en Proyectos
}

\author{
Carlos A. Collazos $^{(1) \star}$, Heindel R. Otero ${ }^{(2)}$, Jaime J. Isaza ${ }^{(2)}$ y César Mora ${ }^{(1)}$ \\ (1) Centro de Investigación en Ciencia Aplicada y Tecnología Avanzada, Instituto Politécnico Nacional, \\ México D.F., México. (e-mail: cacollazos@gmail.com; ceml36@gmail.com) \\ (2) Departamento de Ciencias Naturales, Escuela Colombiana de Ingeniería, Bogotá D. C., Colombia. \\ (e-mail: heindel1297@gmail.com; jisazaceballos@gmail.com) \\ * Autor a quien debe ser dirigida la correspondencia
}

Recibido Sep. 15, 2015; Aceptado Nov. 9, 2015; Versión final Ene. 5, 2016, Publicado Abr. 2016

\begin{abstract}
Resumen
Este trabajo presenta los resultados de una estrategia pedagógica fundamentada en el Aprendizaje Basado en Proyectos que fue aplicada en cursos de Electricidad y Magnetismo con estudiantes de Ingeniería de la Escuela Colombiana de Ingeniería en el primer semestre de 2015. Se pidió a los estudiantes que construyeran prototipos de bajo costo que les permitieran involucrar conceptos de modelamiento físico y la teoría de error. La metodología empleada estimula y aumenta el aprendizaje de la electrostática así como también otras habilidades tales como expresión gráfica, oral y escrita. En este trabajo se describe el método usado y los instrumentos que se emplearon para el proceso de obtención de datos. Los análisis muestran una ganancia de $(0.78 \pm 0.03)$ y $(0.65 \pm 0.04)$ para la evaluación de los proyectos y $(0.47 \pm 0.04)$ para una prueba conceptual de electrostática. El trabajo presenta y discute las fortalezas y dificultades de la estrategia empleada.
\end{abstract}

Palabras clave: electrostática; construcción; prototipos; aprendizaje; proyectos.

\section{Teaching Electrostatics through the Construction of Low Cost Prototypes and Project Based Learning}

\begin{abstract}
This work presents the results of a teaching strategy based on Projects Based Learning which was applied in a course of Electricity and Magnetism for engineering students of the Colombian School of Engineering in the first semester of 2015. The students were required to construct low cost prototypes that let them to use concepts of physical modeling and error theory. The methodology employed stimulates and increases the learning of electrostatics and of other abilities such as graphical, oral and written expression. This paper describes the method used and the instruments that were used for the data collection process. Analyses show a gain of $(0.78 \pm 0.03)$ and $(0.65 \pm 0.04)$ for the evaluation of the projects and $(0.47 \pm 0.04)$ for an electrostatic conceptual test. The paper presents and discusses the strengths and difficulties of the strategy employed.
\end{abstract}

Keywords: electrostatics; construction; prototypes; learning; projects 


\section{INTRODUCCIÓN}

Los programas a nivel de Física involucran a la Electrostática dentro de su currículo educativo, sin embargo no solo se requiere de la acumulación y verificación de conceptos como a veces sucede, sino también del desarrollo de habilidades que formen al estudiante para el análisis y la resolución de problemas. Entre las estrategias a emplear se encuentran, trabajos de semestre o también llamados trabajos prácticos donde la comprensión de los fenómenos físicos se desarrolla por medio de la ejecución de proyectos como se indica en (Castellanos y D'Alessandro, 2003; Barbosa et al., 2011; Collazos et al., 2014; Valderrama y Valderrama, 2014). El presente artículo se identifica teóricamente con algunos aspectos del constructivismo y de forma más particular con el Aprendizaje Basado en Proyectos. La instrucción basada en proyectos sugiere actividades de enseñanza interdisciplinar, de largo o mediano plazo y enfocada en el estudiante, en lugar de procesos educativos cortos y aislados. El Aprendizaje Basado en Proyectos tiene su origen en la aproximación constructivista que evolucionó a partir de los trabajos de psicólogos y educadores como Lev Vygotsky, Jerome Bruner, Jean Piaget y John Dewey (Hernández, 2004). La construcción y experimentación con los prototipos bajo un ambiente de Aprendizaje Basado en Proyectos permite involucrar al estudiante y profesor en un ciclo experimental (predicción, observación y validación) el cual tiene directa relación con el método científico (Hernández, 2004). Este trabajo se enfoca en dos proyectos que son: Electroscopio (P1) y Generador de Van de Graaff (P2). Los proyectos han sido ejecutados para el primer periodo de 2015 con estudiantes que pertenecen a Facultades de Ingeniería de la Escuela Colombiana de Ingeniería. El trabajo se ha estructurado de la siguiente manera: en la sección Metodología se plantea el objetivo, justificación, pregunta de Investigación e Hipótesis de este trabajo. En la sección Fundamento Teórico se hace una revisión al aprendizaje basado en proyectos y se presenta la estrategia de construcción de prototipos en la Electrostática. En la sección Resultados presentamos los análisis a nivel de la evaluación por proyectos y la evaluación conceptual (prueba tradicional). En la sección Conclusiones se presentan las fortalezas y dificultades de la estrategia empleada a nivel de los estudiantes.

El objetivo de este trabajo es medir y valorar cómo las estrategias de Construcción de Prototipos y el Aprendizaje Basado en Proyectos aumentan la eficacia en la enseñanza de la electrostática a nivel de un curso de Electricidad y Magnetismo. De igual forma determinar pertinencia, eficiencia y falencias en el aprendizaje de los estudiantes. El trabajo se justifica por varias razones: i) Introducir una conexión entre la construcción de prototipos y el Aprendizaje Basado en Proyectos en la enseñanza de un curso de Electricidad y Magnetismo a nivel universitario. Aunque existen evidencias del uso de prototipos en la enseñanza de la física, como por ejemplo en las publicaciones American Journal Physics, The Physics Teacher, Latin American Journal Physics Education y Revista Brasilera de Ensino Física, hay muy poco material pedagógico relacionado con el ciclo de aprendizaje en el que está inmerso un estudiante cuando construye y experimenta con un prototipo en electrostática; ii) Obtener resultados cuantitativos y cualitativos derivados de la aplicación de la metodología basada en proyectos. Al aplicar la metodología a la Construcción de Prototipos, se observó su utilidad para percibir y suprimir falsos conceptos afines a la Fuerza y Campo Eléctrico, formas para cargar eléctricamente un cuerpo, modelamiento físico, teoría de error y análisis gráfico. Así mismo se pudieron obtener otros resultados en cuanto a otros aprendizajes relacionados con la expresión oral, escrita y gráfica; y iii) Implementar instrumentos de medida entorno a: el contexto en el que se puede aplicar la estrategia dentro de un periodo académico, además de la iniciativa y motivación de los estudiantes.

La hipótesis de trabajo que sustenta la propuesta es que el Aprendizaje Basado en Proyectos y la Construcción de Prototipos presentan una ganancia en la efectividad de la enseñanza de La Electrostática en comparación con la Instrucción tradicional, ya que permite al instructor diseñar y aplicar por un lado estrategias de trabajo experimental (teoría de error y análisis gráfico) y por otro, estrategias de trabajo teórico (modelamiento físico), además que también genera otros aprendizajes a nivel de expresión oral, escrita, grafica, habilidades experimentales y trabajo en grupo.

\section{FUNDAMENTO TEORICO}

La metodología del Aprendizaje Basado en Proyectos parte de generar tareas complejas a partir de preguntas difíciles o de problemas que implica a los estudiantes resolverlos, tomar decisiones e involucrar habilidades de investigación en colaboración con el maestro. La metodología se centra en aspectos que conducen a los estudiantes a encontrar los conceptos fundamentales y principios de un tema práctico. Los estudiantes forman su propia investigación de una pregunta orientadora, lo que les permite desarrollar habilidades valiosas como el trabajo en equipo. Los estudiantes participan en el diseño, la resolución de problemas y toma de decisiones.

Los aspectos más relevantes de la metodología basada en el Aprendizaje Basado en Proyectos según Benítez y García (2012) son: i) Centrados en el estudiante. ii) Definidos, un inicio, un desarrollo y un final. iii) 
Contenido significativo para los estudiantes directamente observable en su entorno. iv) Se abordan problemas definidos. Los beneficios son: i) Preparará a los estudiantes para la vida profesional y laboral. ii) Incrementa la motivación en estudiantes y profesores. iii) Establece una conexión entre el aprendizaje en la universidad y la realidad. iv) Aumenta las habilidades sociales y de comunicación. La estructura es i) Problema: Se debe describir el tema o problema que el proyecto busca atender o resolver; ii) Descripción y propósito del proyecto: Una explicación concisa de los objetivos del proyecto y de qué manera atiende éste la situación o el problema; iii) Especificaciones de desempeño: Lista de criterios o estándares de calidad que el proyecto debe cumplir. iv) Reglas: Guías o instrucciones para desarrollar el proyecto. Incluye tiempo presupuestado y metas a corto plazo, listado de los participantes en el proyecto con los roles que se les asignaron; y v) Evaluación: Cómo se va a valorar el desempeño de los estudiantes. En el aprendizaje por proyectos, se evalúa tanto el proceso de aprendizaje como el producto final. Es habitual en este tipo de proyectos incluir criterios de evaluación definidos de antemano por medio de Rúbrica.

\section{LA CONSTRUCCIÓN DE PROTOTIPOS}

La Construcción de Prototipos es una estrategia fundamentada en el Aprendizaje Basado en Proyectos que implica la integración de conocimientos a nivel de la Electrostática. Cuando un estudiante construye un prototipo dimensiona la física como una ciencia que le permite crear, ingeniar, analizar y resolver problemas; además le permite activar otras habilidades a nivel de expresión gráfica, oral y escrita y usos de la tecnología existente. Para planificar el uso de prototipos en la enseñanza de la Electrostática hemos revisado las publicaciones American Journal Physics y The Physics Teacher. Los principales artículos y los tópicos afines a este trabajo son: i) Carga, fuerza y campo en electrostática: (Hassan,1995; Price and Phillips, 1990) y ii) Máquinas y Prototipos en Electrostática: (Heering, 1992; Xie and Huang ,1994). En la figura 1 se indican algunos de los prototipos desarrollados por los estudiantes. El procedimiento a seguir con los prototipos se centra en: i) Trabajar experimentalmente entorno a preguntas y cuestionamientos. ii) Involucrar el ciclo experimental (predicción, observación y validación). iii) Generar en el estudiante capacidades alrededor del análisis gráfico y de la teoría de error para la validación de los fenómenos observados. Un mayor detalle de los prototipos puede ser encontrado en fisicacollazos, 2015.

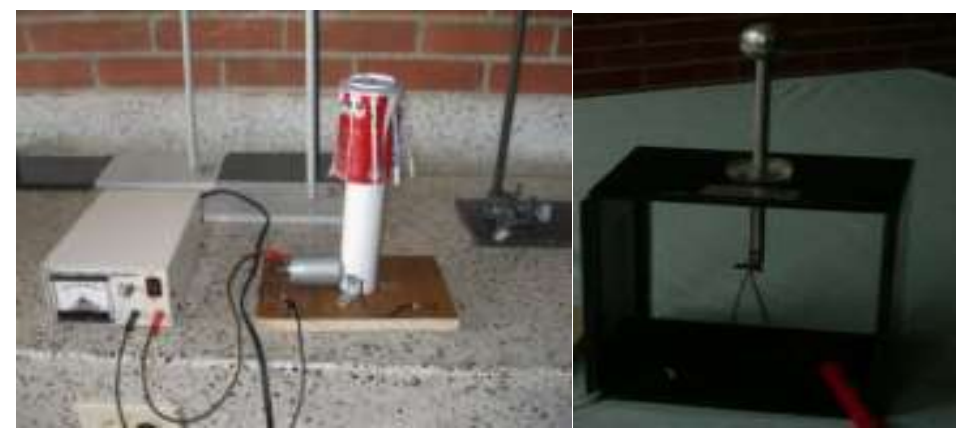

Fig. 1. Prototipos construidos por estudiantes (Generador de Van der Graaff y Electroscopio).

\section{Actividades guiadas durante un semestre académico}

La estrategia inicia con una introducción en la primera semana de clases; para esta instancia se definen los subgrupos (3 estudiantes). Cada grupo de estudiantes abordan los dos proyectos: Electroscopio (P1) y Generador de Van de Graaff (P2). En la primera semana los estudiantes conocen las reglas fundamentales bajo las cuales se va a definir, ejecutar y presentar los proyectos. También en la primera semana se aplica una prueba conceptual de entrada (prueba diagnóstica), $\left(\mathrm{S}_{\mathrm{i}}\right)$. En la tercera semana los estudiantes hacen entrega escrita de su anteproyecto y realizan la presentación oral I; estas dos actividades determinan la evaluación 1 (Eva-1). Una semana después se realizó la retroalimentación a cada uno de los proyectos, donde se indica qué propuestas son viables y cuáles deben replantearse. En la cuarta semana, se orienta el Taller I (Expresión Oral y Escrita) en cuanto a presentaciones orales, informes escritos y artículos. En la sexta semana se orienta un Taller II (Expresión Gráfica) en cuanto al diseño y la construcción de prototipos en ingeniería (Normas Técnicas para el levantamiento de planos, materiales etc.). Para la octava semana y con base a las recomendaciones hechas hasta el momento los estudiantes deben adecuar su prototipo para la experimentación. En esta semana los estudiantes realizan el proceso experimental (predicción, observación y validación) con el prototipo. Los estudiantes tienen la novena semana para ajustes de último momento. En la semana 10, se realiza la entrega escrita del avance-1 y la presentación oral II. Estas dos actividades determinan la evaluación 2 (Eva-2). En la semana 11 se indican las fortalezas y debilidades que tiene el proyecto. En la semana 12 se orienta el Taller III: Modelamiento Físico, Teoría de error y Análisis gráfico. Así mismo se indican los principales tipos de modelos y su interpretación con base en los prototipos desarrollados y en el análisis de datos experimentales; En la semana 14 se repite el ciclo experimental 
(predicción, observación y validación) con los prototipos reforzando la teoría de error y el análisis gráfico. En la semana 16 se realiza la presentación oral III y la entrega escrita de un artículo en formato científico; estas dos actividades determinan la evaluación 3 (Eva-3), además se aplica nuevamente la prueba diagnóstica (prueba final), $\left(\mathrm{S}_{\mathrm{f}}\right)$. Para mayor detalle el material de la metodología empleada está disponible en fisicacollazos, 2015.

\section{Población de Estudiantes}

La estrategia se aplicó a los estudiantes de tercer semestre de la Facultad de ingeniería de la Escuela Colombiana de Ingeniería (ECI) en Bogotá-Colombia. En este semestre los estudiantes pertenecen al periodo de formación en Ciencias Básicas y coinciden en materias como Física de Electricidad y Magnetismo y Cálculo Integral. Los estudiantes pertenecen a los programas de Ingeniería Electrónica, Eléctrica, Civil, Biomédica, Mecánica, Sistemas, e Industrial. La estrategia pedagógica fue aplicada dentro del curso de Electricidad y Magnetismo a 4 grupos (Grupos Experimentales) que se conforman de 120 estudiantes. Cada grupo se compone por 30 estudiantes. Posteriormente se formaron Subgrupos de 3 estudiantes. En total Se consolidaron 40 subgrupos. Adicionalmente existen otros 4 grupos (Grupos de Control) del curso de Electricidad y Magnetismo conformado por 120 estudiantes a los cuales no se aplicó la estrategia, es decir que este último tipo de grupos corresponde a grupos de instrucción tradicional donde los estudiantes no desarrollaron proyectos ni construyeron prototipos. Los grupos de control tienen una intensidad horaria semanal de 4.5 horas de clase teórica y 1.5 horas de laboratorio. La temática de electrostática se cubre con 4.5 horas (sesión de teoría) y 1.5 horas (sesión de laboratorio).A los grupos experimentales se les aplicó tanto la evaluación por proyectos ((Eva-1), (Eva-2) y (Eva-3)) como la evaluación conceptual $\left(S_{i}\right.$ y $\left.S_{f}\right)$. En los grupos de control solo se aplicó evaluación conceptual $\left(\left(S_{i}\right)\right.$ y $\left.\left(S_{f}\right)\right)$.

\section{Evaluación}

La estrategia empleada tienes dos tipos de evaluación, una es la evaluación por proyectos ((Eva-1), (Eva-2) y (Eva-3)) y la otra es la Prueba conceptual de selección múltiple, $\left(\mathrm{S}_{\mathrm{i}}\right)$ y $\left(\mathrm{S}_{\mathrm{f}}\right)$. La evaluación por proyectos es grupal mientras que la Prueba conceptual de selección múltiple es individual. A continuación se describe cada una de las evaluaciones. a) (Eva-1): Definición de la propuesta: anteproyecto y presentación oral I, se realizó en la semana 3 teniendo un valor 25\%; b) (Eva-2): Fundamentación del proyecto: avance 1 y presentación oral II, se realizó en la semana 10 teniendo un valor 35\%; y c) (Eva-3): Resultados Finales: entrega de artículo y presentación oral III, se realizó en la semana 16 con un valor 40\%. Las tres evaluaciones se califican sobre 100 puntos y los estudiantes pueden conocer con anticipación los criterios de evaluación como se indica en la Tabla 1. Estos criterios describen varios niveles de calidad (Excelente, bueno, regular, pobre y nulo) y el propósito es hacer una retroalimentación continua con los estudiantes sobre la evolución en cada una de las actividades planteadas. Las categorías de aprendizaje incluidas en la rúbrica son Expresión oral, Expresión Escrita, Expresión Gráfica, Ciclo Experimental y Retroalimentación y están detalladas en fisicacollazos, 2015.

Tabla 1. Criterios de Evaluación: E:Excelente; B: Bueno; R: Regular; P:Pobre; N: Nulo

\begin{tabular}{|l|c|c|c|c|c|c|}
\hline (Eva-1),(Eva-2);(Eva-3) & Valor & N & P & R & B & E \\
\hline 1. Expresión Oral & 20 & 0 & 4 & 11 & 16 & 20 \\
\hline 2. Expresión Escrita & 20 & 0 & 4 & 11 & 16 & 20 \\
\hline 3. Expresión Gráfica & 20 & 0 & 4 & 11 & 16 & 20 \\
\hline 4. Ciclo Experimental & 30 & 0 & 5 & 11 & 25 & 30 \\
\hline 5. Retroalimentación & 10 & 0 & 2 & 6 & 8 & 10 \\
\hline
\end{tabular}

La evaluación Conceptual es una evaluación tradicional que consta de 30 preguntas de electrostática donde se evalúa tradicionalmente principios generales de la electrostática (fricción, inducción y conducción), conceptos de fuerza y campo eléctrico. Las preguntas fueron extractadas de (Riley, 2011). El cuestionario se aplica en la primera semana $\left(S_{i}\right)$ y la última semana de clases $\left(S_{f}\right)$. A manera de ejemplo la Tabla 2 presenta la pregunta número 4 del cuestionario. La prueba completa con su solución puede ser descargada en fisicacollazos, 2015.

Tabla 2. Pregunta No. 4 de Prueba Conceptual

4) Dos pequeñas esferas se atraen electrostáticamente. Esto puede ocurrir por una variedad de razones. ¿Cuál de las siguientes afirmaciones es verdadera?

A) Al menos una de las esferas está cargada. D) Las esferas tienen la misma carga.

B) Ambas esferas están cargadas. E) Ninguna de estas respuestas es correcta.

C) Ninguna de las esferas está cargada. Respuesta: A 


\section{RESULTADOS}

Las Tabla 3 y 4 indican los resultados promedio $\mu$ y su respectiva desviación estándar $\sigma$ para los 4 grupos experimentales en el proyecto (P1) y proyecto (P2) respectivamente. Las Tablas 3 y 4 indican que los estudiantes comienzan con un desempeño regular en la primera evaluación (Eva-1) y que corresponde a un valor de $(56.04 \pm 3.20)$ para $(P 1)$ y de $(43.30 \pm 3.96)$ para $(P 2)$.

Tabla 3. Resultados promedio para criterios de evaluación de (P1) para los 4 grupos experimentales

\begin{tabular}{|l|c|c|c|c|}
\hline \multicolumn{1}{|c|}{ Proyecto (P1) } & Valor & $\mu$ (Eva-1) $\pm \sigma$ (Eva-1) & $\mu$ (Eva-2) $\pm \sigma$ Eva- & $\mu$ (Eva-3) $\pm \sigma$ (Eva-3) \\
\hline 1. Expresión Oral & 20 & $11.25 \pm 3.16$ & $12.40 \pm 2.38$ & $13.42 \pm 3.79$ \\
\hline 2. Expresión Escrita & 20 & $12.77 \pm 2.42$ & $14.85 \pm 2.89$ & $16.00 \pm 1.75$ \\
\hline 3. Expresión Gráfica & 20 & $12.31 \pm 2.59$ & $15.83 \pm 1.77$ & $16.42 \pm 1.93$ \\
\hline 4. Ciclo Experimental & 30 & $13.63 \pm 3.52$ & $24.29 \pm 3.46$ & $25.92 \pm 2.70$ \\
\hline 5. Retroalimentación & 10 & $6.08 \pm 1.09$ & $7.29 \pm 1.20$ & $7.50 \pm 3.21$ \\
\hline 6. Consolidado Total & 100 & $56.04 \pm 3.20$ & $74.67 \pm 2.83$ & $79.26 \pm 3.00$ \\
\hline
\end{tabular}

Tabla 4. Resultados promedio para criterios de evaluación de (P2) para los 4 grupos experimentales

\begin{tabular}{|l|c|c|c|c|}
\hline \multicolumn{1}{|c|}{ Proyecto (P2) } & Valor & $\mu$ (Eva-1) $\pm \sigma$ (Eva-1) & $\mu$ (Eva-2) $\pm \sigma$ Eva- & $\mu$ (Eva-3) $\pm \sigma($ Eva-3) \\
\hline 1. Expresión Oral & 20 & $9.40 \pm 4.02$ & $10.71 \pm 3.90$ & $12.88 \pm 4.18$ \\
\hline 2. Expresión Escrita & 20 & $10.58 \pm 3.72$ & $11.29 \pm 3.70$ & $12.04 \pm 3.85$ \\
\hline 3. Expresión Gráfica & 20 & $10.17 \pm 3.86$ & $10.56 \pm 3.71$ & $12.75 \pm 4.01$ \\
\hline 4. Ciclo Experimental & 30 & $10.04 \pm 4.04$ & $17.63 \pm 4.12$ & $19.81 \pm 3.90$ \\
\hline 5. Retroalimentación & 10 & $3.04 \pm 3.80$ & $5.38 \pm 4.18$ & $8.10 \pm 4.40$ \\
\hline 6. Consolidado Total & 100 & $43.30 \pm 3.96$ & $55.67 \pm 4.36$ & $65.94 \pm 3.98$ \\
\hline
\end{tabular}

Puede notarse en la Tabla 3, que en la evaluación intermedia (Eva-2) hay una mejora progresiva que corresponde a un valor de $(74.67 \pm 2.83)$ para (P1) y $(55.67 \pm 4.36)$ para (P2). En la evaluación final (Eva-3) se observa que los estudiantes terminan el proyecto con un desempeño adecuado con un valor de $(79.26 \pm 3.00)$ para $(\mathrm{P} 1)$ y $(65.94 \pm 3.98)$ para $(\mathrm{P} 2)$.

Con base al consolidado total de las Tablas 3 y 4, procedimos a utilizar la ecuación de ganancia que fue usada por (Hake, 1999). La ganancia es un indicador cuantitativo de uso actual en la enseñanza de la Física como se indica en (GS, 2015). Este indicador compara una prueba de salida con una de entrada. La ganancia está asociada a un valor numérico entre 0 y 1 . La ganancia se clasifica en ideal $\left(g_{31}=1\right)$, alta $\left(1>g_{31}>0.7\right)$, (media $\left(0.7>g_{31}>0.3\right)$, y baja $\left(0.3>g_{31}>0\right)$. De esta manera se procedió a determinar la ganancia $g_{31}$ entre (Eva-1) y (Eva-3) por la siguiente ecuación:

$g_{31}=\frac{(\text { Eva-3) }-(\text { Eva-1) }}{100-(\text { Eva-1) }}$

La Figura 2 presenta la ganancia promedio $\bar{g}_{31}$ y su respectiva desviación estándar $\sigma_{g_{31}}$ para los 4 grupos experimentales en los proyectos (P1) y (P2). Se observa un rango ganancia de $(0.78 \pm 0.03)$ para (P1) y de $(0.65 \pm 0.04)$ para $(P 2)$. De acuerdo a los resultados se observa cambios significativos entre las evaluaciones (Eva-1) y Eva-3), las cuales fueron aplicadas antes y después de emplear la estrategia pedagógica a nivel de los criterios de evaluación: expresión oral, expresión escrita, expresión gráfica, ciclo experimental y retroalimentación. Los cambios fueron cuantificados y se observó una ganancia alta entre 0.7 y 1 para (P1) y una ganancia media entre 0.3 y 7 para (P2). Estos cambios reflejan que los estudiantes tienen mejores argumentos para sustentar y escribir sus resultados en la medida que el proyecto avanza, además muestran mejoras progresivas en el diseño, construcción y experimentación con cada uno de los prototipos. 


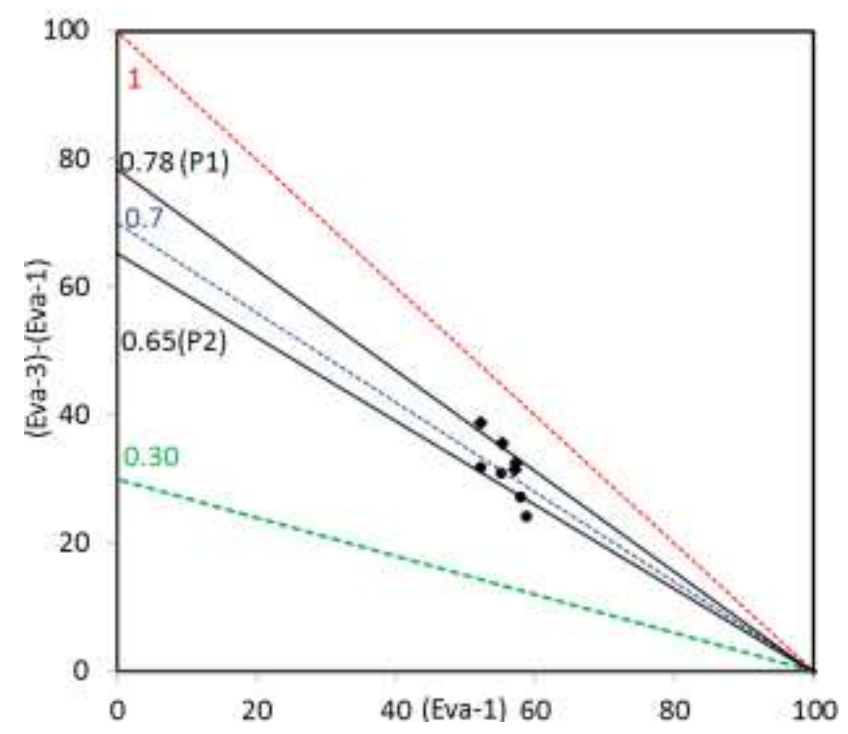

Fig. 2: Dispersión de grupos experimentales: $\overline{\mathrm{g}}_{13}=0.78, \sigma_{\mathrm{g}_{13}}=0.03$, en Electroscopio (P1) y $\bar{g}_{13}=0.65, \sigma_{g_{13}}=0.04$, en Generador de Van de Graff (P2).

Empleando el concepto de ganancia definido anteriormente y ahora readaptado por la siguiente ecuación:

$g_{f i}=\frac{S_{f}-S_{i}}{100-S_{i}}$

$S_{i}$ y $S_{f}$ corresponden respectivamente a los resultados de la prueba conceptual antes y después de aplicar la estrategia para el caso de los grupos experimentales. La prueba también fue aplicada a los grupos de control, los cuales son grupos donde no se aplicó la estrategia. En la Figura 3 se observa un rango de ganancia media de $(0.47 \pm 0.04)$ para los grupos experimentales superior a 0.3 e inferior a 0.7 . Para los grupos control superior a 0 e inferior a 0.3 se obtuvo un rango ganancia baja de $(0.19 \pm 0.06)$. Los resultados indican que el desempeño de los grupos experimentales es superior respecto a los grupos de control. La superioridad puede obedecer al refuerzo empírico y conceptual que tiene los estudiantes de los grupos experimentales al desarrollar proyectos y construir prototipos. La ganancia baja obtenida en los grupos de control puede ser ocasionada en parte por el uso de un modelo tradicional de enseñanza, el cual se limita en algunas ocasiones a transmitir y comprobar conceptos. Es importante resaltar que la electrostática para los estudiantes e incluso algunos profesores tiene complejidad por los tópicos involucrados durante el proceso enseñanza-aprendizaje. Principios como la cuantificación y la conservación de la carga, mecanismos para producción de cargas (fricción, inducción), tipos de materiales (conductores, aislantes) y el carácter vectorial de la fuerza y el campo eléctrico requieren de estrategias pedagógicas adicionales a la instrucción tradicional para que los estudiantes conceptualicen adecuadamente la electrostática(Collazos et al., 2014 ).

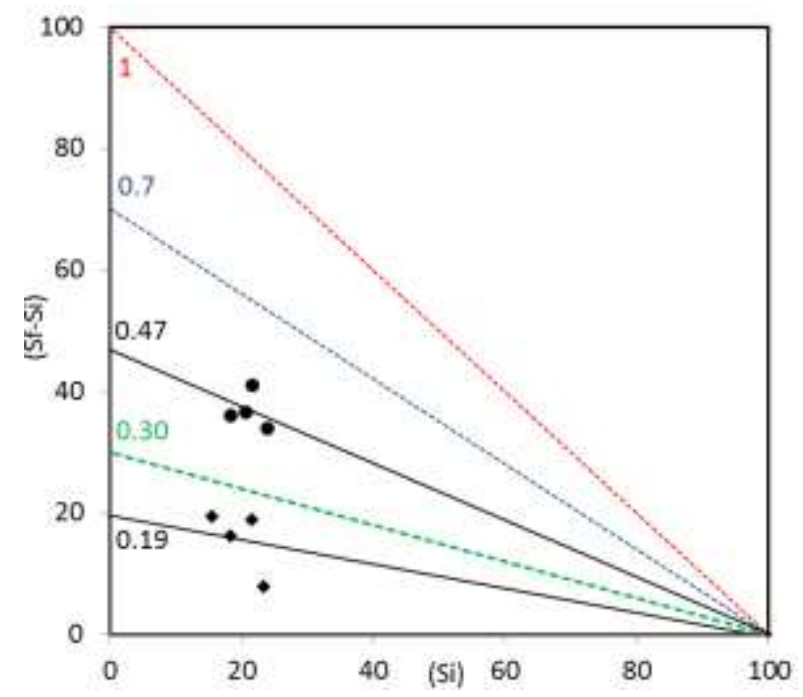

Fig. 3: Dispersión de grupos: $\overline{\mathrm{g}}_{13}=0.47, \sigma_{\mathrm{g}_{13}}=0.04$, en grupos experimentales y $\bar{g}_{13}=0.19 ; \sigma_{\mathrm{g}_{13}}=0.06$, en grupos de control. 


\section{CONCLUSIONES}

La estrategia del Aprendizaje Basado en Proyectos y la Construcción de Prototipos muestra un rango de ganancia medio para los grupos experimentales en la prueba conceptual de electrostática. De esta manera se comprueba la hipótesis planteada en la Introducción, acerca de que la metodología empleada en este trabajo presenta una ganancia respecto a la instrucción tradicional(grupos de control), donde se obtuvo un rango de ganancia bajo.

Se observa cambios significativos para la evaluación de proyectos a nivel de los criterios de evaluación: Expresión oral, Expresión Escrita, Expresión Gráfica, Ciclo Experimental y Retroalimentación. Se obtuvieron rangos de ganancia alto para (P1) y medio para (P2). De esta forma se comprueba la hipótesis acerca de que la estrategia empleada genera aprendizajes no solo en física sino de expresión oral, escrita, gráfica y habilidades experimentales de trabajo en equipo.

En general el esquema de análisis de resultados permite medir cuantitativamente y valorar cualitativamente la estrategia empleada. Se pudo establecer que los estudiantes:

- Construyen prototipos que les permiten conceptualizar adecuadamente los tópicos de electrostática como inducción, fricción, conducción y conceptos de fuerza y campo eléctrico.

- Identifican y corrigen en algunos casos falsos conceptos relacionados con los principios fundamentales de la electrostática como son la conservación y la cuantificación de la carga, además del concepto de tierra y portadores de carga.

- Trabajan activa y colaborativamente en la ejecución de los proyectos. La estrategia llevó a los estudiantes a desarrollar una consulta acerca del tema planteado, para ello realizaron la revisión de diferentes fuentes (páginas en la web, libros, revistas de divulgación científica, consultas a expertos, entre otros). Luego seleccionaron la información que les permitió solucionar el proyecto formulado.

- Pudieron establecer un proceso de experimentación (predicción, observación y validación) con los prototipos desarrollados. Aunque los estudiantes manifiestan que la teoría de error y el análisis gráfico les generan en principio dificultad y confusión, al finalizar el proyecto realizaron un buen análisis e interpretación de los datos experimentales obtenidos.

- Construyeron y modelaron físicamente el prototipo. Para ello usaron métodos de razonamiento inductivo y deductivo que les permitió identificar las variables físicas involucradas como también definir variables dependientes, independientes y constantes. Igualmente se realizaron medidas de dichas variables para poder hacer los cálculos correspondientes. En este sentido la solución teórica y práctica del proyecto fortaleció las habilidades investigativas, tales como: observar, analizar, deducir, conjeturar, ingeniar, producir, entre otras.

-Reconocieron que las temáticas afines a los proyectos no son sencillas, sin embargo resaltan que el contexto en el que se desarrollan los proyectos permite tener una mejor profundización conceptual en los temas de Electrostática. El diseñar y construir los prototipos, les permitió lograr una apropiación y aplicación de los conceptos de electrostática; fortalecieron su nivel de abstracción, en tanto que el estudiante pudo concebir la conceptualización física como una herramienta que le permite dar solución a una problemática planteada.

- Manifestaron que las presentaciones orales y escritas son de gran importancia ya que los prepara dentro de su quehacer cotidiano como estudiantes de ingeniería. Del mismo modo, el diseño y construcción de prototipos involucra el desarrollo de habilidades y destrezas técnicas, manifestadas en el aspecto experimental que permitió el funcionamiento del mismo. Los proyectos también lograron sensibilizar a los estudiantes, evidenciando valores, tales como: autoestima, automotivación, trabajo en grupo, entre otros.

\section{REFERENCIAS}

Barbosa L. H., Mora C., Talero P., Organista J. O., El Soplador mágico: un experimento discrepante en el aprendizaje de la ley de presión hidrodinámica de Bernoulli, doi: 10.1590/S1806-11172011000400009, Revista Brasileira de Ensino de Física (en línea), 33(4), 1-7 (2011)

Benitez A. A., Garcia M. L., Un Primer Acercamiento al Docente frente a una Metodología Basada en Proyectos, doi: 10.4067/S0718-50062013000100004, Formación Universitaria (en línea), 6(1), 21-28 (2013) 
Castellanos, M.L. y A. D'Alessandro, Proyectos de Investigación: Una Metodología para el Aprendizaje Significativo de la Física en Educación Media, Rev. Pedagogía, ISSN 0798-9792 (en línea): 24(69), 101-136 2003,http://www.scielo.org.ve/scielo.php?script=sci_abstract\&pid=S079897922003000100005\&lng=es\&nrm =iso\&tlng=es. Acceso 15 de diciembre (2015)

Collazos, C. A., R. Otero, Isaza J. y Mora C., Teaching Of Physics Throught Of The Construction Of Prototypes, The GIREP-MPTL: International conference on Teaching/Learning Physics: Integrating Research into Practice, Palermo, Italia (2014)

Collazos, C. A., fisicacollazos, Página Web de Carlos Collazos, http://www. fisicacollazos.260mb.com/?cat=46. Acceso: Diciembre (2015)

SG, Scholar Google, https://scholar.google.com/citations?user=10El2q8AAAAJ\&hl=en, acceso Diciembre (2015)

Hake, R., Interactive-engagement vs traditional methods: A six-thousand-student survey of mechanics test data for introductory physics courses, doi: 10.1119/1.18809, American Journal Physics (en línea), 66, 64-74 (1996)

Hassan H., Comment on "Elegant calculations of the Coulomb force between two hemispherical surfaces with uniform charge densities" by Xishun Xie and Xiaogin Huang [AJP 62, 952-953 (1994)], doi: 10.1119/1.17814, American Journal Physics (en línea),63, 856 (1995)

Heering P., On Coulomb's inverse square law, doi: 10.1119/1.17002, American Journal Physics (en línea), 60, 988-994 (1992)

Hernández, C., Aprendizaje de la Física en estudiantes de diseño Industrial dentro de una innovación pedagógica consistente en el constructivismo, Tesis de Maestría en Educación, Universidad de Los Andes, Bogotá, Colombia (2004)

Price R. and Phillips R., The Force Between Two Charged Wires, doi: 10.1119/1.16443, America Journal Physics (en línea), 58, 534-539 (1990)

Riley M., Test Bank of Physics, Ed. W. H. Freeman and Company, New York (NY), USA (2011)

Valderrama A. J., Valderrama J. O., Trabajo Semestral Guiado: un Buen Complemento al Estudio y Aprendizaje, doi: 10.4067/SO71850062014000300002, Formación Universitaria (en línea), 7(3), 3-10 (2014)

Xie X. y Huang X. Elegant calculations of the Coulomb force between two hemispherical surfaces with uniform charge densities, doi: 10.119/1.17691, American Journal Physics (en línea), 62, 952-953 (1994) 